\section{ONCOCYTIC MUCOEPIDERMOID CARCINOMA: A CASE REPORT AND REVIEW OF LITERATURE}

$\underline{\text { Sevastjan Kranz }}$

Department of Anatomical Pathology, Royal Melbourne

Hospital, Parkville, Vic, Australia

Case presentation: A 64-year-old man presented with a one week history of firm right parotid swelling. Computed tomography $(\mathrm{CT})$ and magnetic resonance imaging (MRI) confirmed a right parotid mass with possible infiltration into preauricular soft tissue and the zygomatic arch. The patient proceeded to a radical right parotidectomy with segmental resection of temporal bone. Histology revealed an infiltrative tumour arising from the parotid comprising cystic spaces and cribriform glands lined predominantly by oncocytes with few mucocytes. Tumour cells showed diffuse staining with p63 and mucocytes stained with PAS and Alcian blue. A diagnosis of oncocytic variant of mucoepidermoid carcinoma (MEC) was made.

Discussion: Oncocytic mucoepidermoid carcinoma (OMEC) is a rare variant of MEC composed chiefly of oncocytic cells. Recognition of this variant is important due to the histologic overlap with other oncocytic salivary gland tumours including benign entities, thus correct diagnosis is essential for appropriate treatment. The presence of typical MEC features such as mucocytes along with diffuse staining of p63 and detection of MAML2 rearrangements helps distinguish OMEC from its mimics. While few cases have been described, OMEC appears to have a favourable prognosis with a low local recurrence rate and minimal propensity for metastasis.

\section{References}

1. Weinreb I, Seethala R, Perez-Oderonez B, et al. Oncocytic mucoepidermoid caricinoma: clinicopathologic description in a series of 12 cases. Am J Surg Pathol 2009; 43: 409-16.

2. Garcia JJ, Hunt JL, Weinreb I, et al. Fluorescence in situ hybridization for detection of MAML2 rearrangements in oncocytic mucoepidermoid carcinomas: utility as a diagnostic test. Hum Pathol 2011; 42: 2001-9.

3. Fujimaki M, Fukumura Y, Saito T, et al. Oncocytic mucoepidermoid carcinoma of the parotid gland with CRTC1-MAML2 fusion transcript: report of a case with review of literature. Hum Pathol 2011; 42: 2052-5.

\section{ANAL SQUAMOUS CELL CARCINOMA IN CROHN'S DISEASE}

Vivienne Lea, Jing Jing Li

Anatomical Pathology, Liverpool Hospital, NSW, Australia

\begin{abstract}
Aim: To present an unusual case of anal squamous cell carcinoma (SCC) arising in the setting of perianal Crohn's disease. Methods: A review of the current literature was undertaken in conjunction with the case report.

Results: A 42-year-old woman with Crohn's disease was admitted with perianal sepsis and underwent a proctocolectomy. On histopathological examination, a well differentiated anal squamous cell carcinoma was found arising within perianal Crohn's disease. The p16 stain was strongly positive in the tumour, suggesting high risk human papilloma virus (HPV) infection as an aetiological factor in addition to the chronic inflammation of Crohn's disease. A review of the literature found anal SCC arising in inflammatory bowel disease is a rare entity. There appears to be a higher incidence of anal SCC and a
\end{abstract}

younger age of presentation in patients with Crohn's disease compared to the general population.

Discussion: The precise aetiology of anal SCC in Crohn's disease, including the role of HPV, remains limited due to the rarity of the condition. Given the increased incidence and younger age of presentation of anal SCC in Crohn's disease, targeted surveillance of longstanding perianal disease should be considered

\section{MUCOEPIDERMOID CARCINOMA OF THE TRACHEA}

\author{
Vivienne Lea, Jim Yong \\ Anatomical Pathology, Liverpool Hospital, NSW, Australia
}

Background: We present an unusual case of low-grade mucoepidermoid carcinoma arising in the trachea.

Case report: A 41-year-old female ex-smoker presented with a one-year history of cough that was investigated by bronchoscopy. A distal tracheal lesion causing $50 \%$ obstruction was identified. Biopsy revealed the lesion to be a low-grade mucoepidermoid carcinoma. The patient underwent tracheal resection and primary anastomosis. This included intra-operative frozen section examination of the proximal and distal margins, which were clear of tumour. Formal histological examination of the resection specimen showed a low-grade mucoepidermoid carcinoma with no mitotic activity or necrosis.

Discussion: Mucoepidermoid carcinomas are uncommon in the tracheobronchial tree. Histological grade and the ability to achieve complete surgical resection are important prognostic factors. Patients with low-grade mucoepidermoid carcinoma generally have a good prognosis after primary surgical resection. In this case, no adjuvant treatment was given, with a plan for ongoing observation with bronchoscopy.

\section{THE UNFOLDED PROTEIN RESPONSE IN BORTEZOMIB RESISTANT MYELOMA CELLS}

Nicholas Nikesitch ${ }^{1,2}$, Chang Tao ${ }^{2,3}$, Ken Lai ${ }^{3,5}$, Murray Killingsworth ${ }^{4}$, Sunna Bae ${ }^{2}$, Silvia C.W. Ling ${ }^{2,3}$

${ }^{1}$ School of Medicine, University of Western Sydney, Campbelltown, ${ }^{2}$ Haematology Research Group, Ingham Institute of Applied Medical Research, ${ }^{3}$ Department of Haematology, Liverpool Hospital, Liverpool, ${ }^{4}$ Electron Microscopy Laboratory, Sydney South West Pathology Service, Liverpool, NSW Health Pathology, and ${ }^{5}$ Cancer Pathology and Cell Biology, Ingham Institute for Applied Medical Research, NSW, Australia

The treatment of multiple myeloma (MM) with novel drugs like proteasome inhibitors (PIs), such as Bortezomib, has improved the survival of most MM patients. The 26S proteasome inhibitor indirectly targets the unfolded protein response (UPR) by inhibiting proteasomal degradation of ubiquitinated paraprotein, subsequently leading to the lethal accumulation of paraprotein within the cell. Initial treatment is effective, however most patients develop resistance. The mechanism of resistance is unknown and the status of the UPR in Bortezomib resistance is unknown. In this project we demonstrate that the UPR is downregulated in Bortezomib resistance. Both in the Bortezomib resistant cell line model and in patients, XBP-1 and ATF6 gene expression is downregulated. Furthermore, the size of the endoplasmic reticulum and the dynamic expansion of the 